\title{
EVALUATING THE EFFECTIVENESS OF INSTRUCTION IN ORAL HYGIENE FOR MENTALLY RETARDED BOYS
}

\author{
By William E. Reynolds, DDS, MPH, ${ }^{*}$ and Robert M. Block, BA**
}

Dr. Reynolds and his group demonstrate that effective toothbrushing can be taught to a group of retarded boys.

Preventive dentistry has received so much attention from both the dental profession and the public during the past few years that several new approaches to dental health education have been developed. The report which follows will describe the results of a two-month, oral-health, educational activity conducted by faculty and students from the University of Michigan. The study included 60 boys between the ages of eight and 13 years at the St. Louis School for Exceptional Boys in Chelsea, Michigan. The investigating group adapted and then applied some of the technics currently advocated for oral health education to a population of mentally retarded boys at a private boarding school. The results obtained by the activity were encouraging and it now is hoped that additional clinical evaluations can be completed with the same group of boys.

\section{Reviewing Some Pertinent Publications}

Although a recent stimulation of interest in oral health education can be detected, little additional evidence has been introduced into the literature to substantiate this resurgence. Clark and Fintz ${ }^{1,2,3}$ and Masters ${ }^{4}$ are proponents of two approaches that they describe as new technics to gain substantially greater success than traditional methods of health education. Both approaches emphasize participation by the students in the learning process rather than the presentation of didactic material by teachers or dental personnel. The program developed by Clark is limited to children in the sixth grade while Masters' program is intended for children in grades one through six. Clark suggests that the instruction be spread throughout the entire school-year after an initial concentrated effort for two weeks. In contrast, Masters' more condensed program is completed after 15 weeks, although it is intended to be repeated in subsequent years.

The role of dental personnel differs to some extent in these two approaches. Masters relies upon dentists from the community to participate in a workshop for teachers which is conducted by representatives of the company which markets the program. Participating dentists instruct the teachers in the procedures of oral hygiene which are included in the program. Instruction about the nature of oral disease and the methods of preventing it also are provided. Masters assumes that the efforts of participating dentists will result in the greatest benefit when they are concentrated on interested teachers. Dental personnel have limited contact with the children because of an opinion that teachers will present the information more effectively.

Clark emphasizes the role of the dentist and dental assistant as teachers. Dentists, assistants, and classroom-teachers all participate in a workshop so that the teaching team will become familiar with the information to be presented. Dental personnel present the information in the classroom and the classroom-teacher acts in a supportive role. Clark expects that the benefits of the instruction will be spread to other members of the

\footnotetext{
* Regional Dental Consultant, Michigan Department of Health, 3500 North Logan Street, Lansing, Michigan 48914; formerly Assistant Professor of Dentistry and Dental Public Health, University of Michigan, Ann Arbor, Michigan

** Senior Dental Student, University of Michigan School of Dentistry, Ann Arbor, Michigan 48104
} 
students' families. His program relies upon mass media in addition to the educational materials taken home by participants.

Both Clark ${ }^{3}$ and Masters ${ }^{5}$ have presented some preliminary evaluatory results to support their claims of success. Clark has completed a 14-month study of approximately 300 children who were divided into an experimental group and a group of controls. He concludes that conventional methods of instruction produce little change in behavior when checked for improved oral hygiene. He finds that his own more comprehensive approach produces a substantial reduction in scores for plaque at the end of eight months, and that this improvement is maintained for six months after activities are terminated in the classroom. Masters ${ }^{5}$ reports the results of a 15-week program completed with a group of 538 children. He finds substantial improvement in gingival health when indexes of gingival inflammation are compared both before and after his program of dental health education. He did not measure long-term benefits and did not have a group of controls.

\section{Method of Study}

St. Louis School was selected for this study because of the degree of concern for oral health exhibited by the School's faculty and the cooperation demonstrated by the School's administration. Enrollment in the School is small. There are four teachers who are certified in special education and four priests who serve as administrators, counselors and instructors. The 60 boys were divided into four classes by age and ability. Age is used as a specific factor in grouping, but ability is correlated more loosely with social and emotional development. Class one is composed of boys, aged eight to 13 years, whose development ranges from the level of preschool to the seventh grade. Boys in class two also are from eight to 13 , but their development ranges from the level of third grade to junior high school. Class three contains boys from 13 to 16 years of age whose development is from preschool through the second grade. The boys in class four also are from 13 to 16 years of age, but their development ranges from third grade through junior high school. All 60 boys participated in the project, but only the 48 present at initial and final assessments of plaque were included in the analysis to be reported.

The School was accessible to the University of Michigan which permitted the investigators to make frequent visits during the period of the study. This project was initiated at the beginning of a semester as a part of an elective course offered by the University's Department of Community Dentistry. Administrative requirements compressed the term of study into a short period. The entire project had to be completed within one semester. During the previous semester senior students of dental hygiene were assigned to St. Louis School one afternoon a week as part of the Department's program of community practice. During this time most of the boys received a prophylaxis, but no formal health education was provided. The enthusiasm of the boys, teachers, and priests in addition to the interest expressed by the students of dental hygiene led to the development of the project to be reported. Mentally retarded children, it was observed, have greater than an average need for oral care, but usually receive minimal treatment only.

The study was designed to utilize to the maximum the positive relationships which exists between teachers and the mentally retarded students. The investigators joined with a public health dental hygientist (health educator) in several meetings with the teachers and priests. These meetings reinforced the importance of oral care and the correct procedure for oral hygiene. After a period of free interchange of ideas a protocol for the study was developed. The scrub method of brushing and the use of disclosing tablets was demonstrated and practiced by the teachers and priests during this period. After two of these seminars, clinical examinations were completed to evaluate the oral hygiene of the teachers and priests. Letters explaining the program of toothbrushing were sent to all of 
the children's parents. No parent refused to allow his child to participate in the toothbrushing.

Available in the health clinic at St. Louis School, were dental chairs with dental lights. Each boy was examined by one of the two dental students who participated in the project while supervised by a member of the faculty of the School of Dentistry. At both the initial and final oral examination, upon which the statistical analysis is based, the students were examined by the same individuals. Each student's teeth were exposed to a staining solution of Bismark brown and plaque was scored by the use of the PHP index. ${ }^{6}$ Sterile gloves, mirrors, and tongue blades were used to examine each student. After the initial score for plaque on each student's teeth was obtained, multi-tuft, youth-sized toothbrushes were issued to each student. Each class of 15 students then was instructed in the correct method of brushing by the dental personnel, the teachers, and the priests. After consultation with the teachers it was agreed that instruction in oral hygiene would not include the use of dental floss. The instruction took place in lighted restrooms in front of a mirror and sink. For the next two days the investigators visited the school and aided in teaching brushing. For the remainder of the study the boys went to their dormitory to brush each day after lunch. During the supervised portion of the study, those boys who needed individual instruction received it from the teachers and priests. The priests sometimes were able to provide some additional attention when the boys were in the dormitory in the morning or the evening. On the seventh day of the study the investigators and the staff at the School dispensed disclosing tablets to each of the students to enable both student and teacher to check their own progress of brushing. This period also was used by the teachers to determine which students were having the most difficulty in obtaining acceptable oral hygiene.

On the fourteenth day another scoring for plaque was completed in the same way as during the original examination. The recorded data on plaque were analyzed to determine progress and a report was supplied to the teachers and priests. This procedure enabled them to select individuals for additional instruction in toothbrushing. Disclosing tablets were supplied to the teachers to dispense to the students at least two times a week for the following six weeks. On the thirty-fifth day, a score for plaque was obtained on each student's teeth.

On the thirty-fifth day, the teachers and priests were told to stop instruction in oral hygiene. They were to continue to provide time for brushing each day after lunch, but no other supervision or encouragement was given on toothbrushing. This period was planned to determine if a habit had been established. On the fifty-sixth day, a final oral examination was performed.

\section{The Results}

The initial scores for plaque were high despite the treatment that had been provided by the students of dental hygiene in the months preceding the study. The mean score for PHP was 3.4 of a possible 5.0 at the first examination. The scores ranged from 3.1 to 3.7 . The improvement in oral hygiene after 14 days of supervised toothbrushing was dramatic. The mean score for PHP dropped to 1.0 with a range of 0.8 to 1.2 . After 45 days, the oral hygiene deteriorated as indicated by a mean PHP of 2.0 with a range of 1.7 to 2.3 . By the time of final examination on the fity-sixth day, oral hygiene had improved, however. The mean PHP at this time was 1.5 with a range of 1.0 to 1.8 . Summaries of these results are presented in Tables I and II.

The mean difference in scores before and after was a reduction of 1.9 with a range of -1.8 to -2.1 . The " $t$ " value for a paired " $t$ " test of all four classes was 11.8 (47 degree of freedom) which is significant at the .001 level. 
Table 1

Mean PHP Scores Before and

After Dental Heal th Education

\begin{tabular}{|c|c|c|c|c|}
\hline \multicolumn{5}{|c|}{$\begin{array}{c}\text { Mean PHP Scores } \\
\text { (Time after initiation of Program in days) }\end{array}$} \\
\hline Class & 1 & 14 & 35 & 56 \\
\hline 1 & 3.7 & 1.2 & 1.9 & 1.8 \\
\hline 2 & 3.5 & .9 & 2.3 & 1.6 \\
\hline 3 & 3.4 & 1.2 & 2.2 & 1.6 \\
\hline 4 & 3.1 & .8 & 1.7 & 1.0 \\
\hline Mean & 3.4 & 1.0 & 2.0 & 1.5 \\
\hline
\end{tabular}

Table II

Differences in PHP Scores Before and After Dental Heal th Education

\begin{tabular}{cccc}
\hline Class & \multicolumn{2}{c}{ Mean PHP Scores } \\
Before & After & Difference \\
\hline 1 & 3.7 & 1.8 & -1.9 \\
2 & 3.5 & 1.6 & -1.9 \\
3 & 3.4 & 1.6 & -1.8 \\
4 & 3.1 & 1.0 & -2.1 \\
Mean & 3.4 & 1.5 & -1.9 \\
\hline \multicolumn{4}{c}{ Paired " $t$ " test for all Four Classes } \\
Significant at the .001 level \\
\multicolumn{4}{c}{$t_{47}=11.8$} \\
d.f. $=47$
\end{tabular}

\section{Some Discussion}

The first scores for plaque showed that the oral hygiene of these boys was poorer than might be expected when compared with children of an average intellectual development. The improvement obtained was particularly dramatic in view of the handicap which the boys experienced. The scores for PHP at the end of the study were comparable to those found by Clark in his sixth-grade controls. These scores still are approximately twice the average score for Clark's experimental group. Clark stated that it was impossible to determine the clinical significance of the reduction that he obtained in his experimental group. A similar question remains in the current investigation.

The investigators had no firm criteria on which to base the timing of evaluation, inasmuch as the decision was made largely because of practical considerations. More than an initial and final examination appeared needed to monitor progress. It was assumed that the boys would have difficulty mastering the technic of toothbrushing and that their skills would improve over a period of time. The results did not confirm this assumption. The boys quickly learned to brush, and their initial enthusiasm produced dramatic results. One boy would not open his mouth for the initial examination. Previously he only had dental care while under general anesthesia. Soon after the program began, he became more cooperative and, by the end of the period, he also was far more receptive to dental care. All classes made posters and bulletin boards in the first several weeks of the program. The boys' interest in oral hygiene became so great that many carried their toothbrushes with them all day, and brushed their teeth while sitting in their classroom.

The third examination demonstrated a return toward their initial status of oral hygiene. Some of the responsibility may have been related to a visit of a group of volunteers at the school the day before the examination in which they presented a bag full of candy and cookies to each boy. When the results of this examination were presented to the teachers, they in turn used this information to motivate the boys. At this point in the study the teachers no longer were supervising the daily brushing, yet there was an improvement at the next examination.

The study answered a number of questions that the investigators had posed during planning. Both the teachers and the investigators were unsure whether any improvement in oral hygiene would be obtained during the course of the study. The teachers were skeptical because their own less formal efforts had not been successful in the past. The teachers were concerned that the boys would not be sufficiently coordinated to learn a method of toothbrushing. They opposed any attempt to teach the use of dental floss for this reason. At the time of the second examination, it was apparent that all of the boys 
had the capability to improve their oral hygiene. The problem that remained was related to motivation as has been found in most other programs of dental health education.

The study also demonstrated that teachers, who are interested in promoting better oral hygiene, can be effective in teaching toothbrushing. It was found important that they receive support and direction from dental personnel.

One might expect that the so-called Hawthorne effect would contribute to the results. The amount of time spent by the dental personnel in visiting the School is likely to have exerted a significant influence. Unfortunately, no opportunity was available to utilize controls because of the small size of the School's population.

\section{Summarizing}

A program of oral health education has been reported at the St. Louis School for Exceptional Boys in Chelsea, Michigan. It was developed because of the School's desire and need for instruciton in oral hygiene. Considerable reliance on the teachers was delegated to provide the motivation for brushing because the mentally retarded boys enrolled at this School presented unique problems. The study, which was completed in eight weeks, included four oral examinations in which the PHP index for scoring plaque was used. The initial examination determined extremely poor oral hygiene. Instruction in toothbrushing then was instituted while major responsibility was assumed by the teachers. Two intermediate examinations were performed, and scores for plaque were tabulated. A final examination at the end of the eight weeks showed a significant reduction in the mean scores. It is important that subsequent studies be completed six months to one year later to check the students' oral hygiene and determine whether the habit of effective toothbrushing twice daily has been established.*

\section{Conclusions}

Three conclusions tentively may be drawn from this study: (1) teachers can be utilized effectively as health educators when they are motivated and dental personnel provide the technical information for correct instruction in oral hygiene; (2) if the mentally retarded child is able to learn the basic skills required to function in a school environment, he should be able to master toothbrushing; and (3) eight weeks were sufficient for these handicapped boys to develop the skills to reduce their scores for plaque significantly.

\footnotetext{
*The assistance of Val Vitols, Senior Dental Student, Dolores Malvitz, MPH, Doctoral Candidate in Dental Public Health, and the Dental Students' Jonathan Taft Society is gratefully acknowledged.
}

\section{Bibliography}

1. Clark, C. A. A manual of dental health; the complete guide for consultant, for teacher, for parent. Cleveland, Greater Cleveland Association Foundation, c1972. V +63 p.

2. Clark, C. A., and Fintz, J. B. A plaque control program for sixth grade children: a preliminary report. Cleveland, Department of Public Health, n.d. 5 p. +3 tables.

3. . And the children shall lead them. Am. Soc. Prevent. Dent. J., 1:27-9, July-Aug.

4. Masters, D. H. The role of the school dental consultant in a dental disease prevention program. Tex. Dent. J., 90:24-7, May 1972.

5. Unpublished data, Aug. 7, 1972.

6. Podshadley, A. G., and Haley, J. V. A method for evaluating oral hygiene performance. Pub. Health Rep., 83: 259-64, Mar. 1968. 